\title{
An Introduction to a Family of Fuzzy Subsets forming Boolean Algebra
}

\author{
Dwiraj Talukdar \\ Department of Information Technology \\ Gauhati University \\ Guwahati-781014, Assam, India
}

\author{
Sisir Kumar Rajbongshi \\ Department of Information Technology \\ Gauhati University \\ Guwahati-781014, Assam, India
}

\begin{abstract}
It has been established that the fuzzy sets do not hold the Complement laws of Boolean algebra. The aim of this article is to introduce and study a kind of family of fuzzy subsets of a finite set which holds the Complement laws. The complement operation has redefined for this purpose. Further, it has been established that the introduced fuzzy subsets can form Boolean lattice and also Boolean algebra.
\end{abstract}

\section{General Terms}

Fuzzy Sets, Boolean algebra, the laws of Boolean algebra.

\section{Keywords}

fuzzy subset, membership value, the complement operation, the complement laws, Boolean lattice, subsets of a fuzzy subset, fuzzy power set.

\section{INTRODUCTION}

In 1965, Lotfi Zadeh [3] first introduced the concept of the fuzzy set theory. The membership in a fuzzy set is the matter of a degree. It indicates that the concept of membership is not always 0 and 1 , but becomes fuzzy which may represent partial belongingness. Due to the important role of fuzzy sets, they have been applied to a large variety of topics in Engineering, Technology and natural and socio economic sciences involving uncertainty, vagueness and ambiguity. They are the powerful mathematical tools for modeling uncertain systems in industry, nature and humanity, in decision making in the absence of precise and complete information $[1,2]$.

Algebraic structures have an important role in Mathematics with applications in Computer Sciences, Information Sciences, and Coding Theory as well as in many other areas. Lattice is an algebraic structure which has wide applications in the theory of design of computers. Lattices are the geometric objects that have been used to solve many problems in Computer Science as well as in Mathematics $[4,8]$.

George Boole, the English Mathematician introduced an algebraic system in 1854 is known as Boolean algebra. The Boolean algebra can also be considered as a special kind of lattice. A bounded, complemented and distributive lattice is known as finite Boolean algebra [5]. Boolean algebra is the Mathematical foundation of switching algebra which is applied to Computer Science and many other areas.

It is accepted that the fuzzy sets cannot form Boolean algebra as it is unable to satisfy all the properties of Boolean algebra. The complement laws are not hold by the fuzzy sets. That is if $\underset{\sim}{A}$ is a fuzzy set and $\underset{\sim}{A^{\prime}}$ is its complement then, $A \cup A_{\sim}^{\prime} \neq$ the universal set, $\underset{\sim}{A \cap} A_{\sim}^{\prime} \neq$ the empty set.
Hence, though the other laws of Boolean algebra are satisfied by fuzzy sets they cannot form Boolean algebra. In this regard, M. Dhar [6] has made an attempt to make the fuzzy sets to hold the complement laws by extending the definition of complementation of fuzzy sets using reference function. This work has been re-visited again in [7] by extending the definition of fuzzy set and proved that the fuzzy sets hold the complement laws.

The aim of this article is to introduce and study a new kind of family of fuzzy subsets of a finite set. The complement operation has redefined and it is established that the introduced fuzzy subsets can hold the complement laws. The observation of the behavior of this family of fuzzy subsets is carried out and proved that they can form a Boolean lattice and hence a Boolean algebra. In support of the definition of complementation lots of numerical examples are illustrated and established that the introduced fuzzy subsets. Further this article introduces new definitions of subset of a fuzzy subset and fuzzy power set of a subset of a fuzzy subset. We have investigated the behavior of such power sets and also established their occurrence in the introduced family of fuzzy subsets of a finite set.

\section{PRELIMINARIES}

Fuzzy sets deal with objects which are "matter-of -degree". It specifies the degree to which an element belongs to the fuzzy set. If $A$ is a classical set of the Universal set $U$, a fuzzy set A is mathematically defined as, $\underset{\sim}{A}=\left\{\left(x, \mu_{\sim}(x)\right): x \in A, \mu_{A}(x) \in[0,1]\right\}$.

Here, $\mu_{A}(x)$ is called the membership function which specifies the grade or degree to which an element $x$ in $A$ belongs to the fuzzy set $\underset{\sim}{A}$.The membership value '1'indicates full membership and ' 0 ' indicates no membership. The fuzzy set containing membership value ' 1 ' for all elements is called the Universal fuzzy set and the fuzzy set containing membership value ' 0 ' for all elements is called the Empty fuzzy set.

If $\underset{\sim}{A}$ and $\underset{\sim}{B}$ are two fuzzy sets defined by-

$$
\begin{aligned}
& \underset{\sim}{A}=\left\{\left(x, \mu_{A}(x)\right): x \in A, \mu_{A}(x) \in[0,1]\right\} . \\
& \underset{\sim}{B}=\left\{\left(x, \mu_{B}(x)\right): x \in B, \mu_{B}(x) \in[0,1]\right\} .
\end{aligned}
$$


Then some basic operations on $A$ and $B$ are defined as follows:-

Inclusion: The fuzzy set $\underset{\sim}{A}$ is said to be included in the fuzzy set $\underset{\sim}{B}$ denoted by $\underset{\sim}{A} \subseteq \underset{\sim}{B}$ if for every $x \in U, \mu_{A}(x) \leq \mu_{\dot{\sim}}(x)$. $\underset{\sim}{A}$ is called the subset of $\underset{\sim}{B}$

Intersection: The intersection of $\underset{\sim}{A}$ and $\underset{\sim}{B}$ is denoted by $A \cap B$ and this operation is mathematically defined as: $\mu_{\sim \sim \sim}^{A \cap B}(x)=\min \left(\mu_{A}(x), \mu_{B}(x)\right), x \in U$.

Union: The union of $\underset{\sim}{A}$ and $\underset{\sim}{B}$ is denoted by $\underset{\sim}{A} \cup \underset{\sim}{B}$ and defined as: $\mu_{\sim} \cup \underset{\sim}{ }(x)=\max \left(\mu_{\sim}(x), \mu_{\underline{\sim}}(x)\right), x \in U$.

Complementation: For any fuzzy set $\underset{\sim}{A}$, its complement is denoted by $A^{\prime}$ and defined as $\mu_{A^{\prime}}(x)=1-\mu_{A}(x), x \in U$.

Difference: The difference between the two fuzzy sets $\underset{\sim}{A}$ and $\underset{\sim}{B}$ is denoted by $\underset{\sim}{A}-\underset{\sim}{B}$ and defined as: $\mu_{A-B}(x)=\min \left(\mu_{A}(x), \mu_{B^{\prime}}(x)\right), x \in U$.

Moreover, the fuzzy sets hold all the laws of Boolean algebra except the complement laws.

\section{INTRODUCTION TO A FAMILY OF FUZZY SUBSETS OF A FINITE SET}

Now, we are going to introduce a family of fuzzy subsets of a finite set. Let $E=\left\{x_{0}, x_{1}, x_{2} \ldots, x_{n-1}\right\}$ is a finite set with $n$ elements. The fuzzy subsets of $E$ are the mappings from $E$ to $I$, where $I=[0,1]$. Let $M$ is the set of the membership values taken by the fuzzy subsets of the finite set $E$,

such

$M=\left\{0, \frac{1}{p}, \frac{2}{p}, \frac{3}{p}, \ldots, \frac{p-1}{p}, \frac{p}{p}=1\right\}$.

$=\{0, h, 2 h, 3 h, \ldots,(p-1) h, p h\}$, where, $h=\frac{1}{p}$

and $p$ is any prime number.

The set $M$ forms a poset under the operation $\leq$ (less than or equal) and also forms a lattice under $\wedge($ gcd $)$ and $\vee(l \mathrm{~cm})$.Then the mapping from $E$ to $M$ contains $|M|^{|E|}$ functions which are nothing but fuzzy subsets. Let the set of all fuzzy subsets of the finite set $E$ with the set $M$ of membership values is denoted by $F$. Now, if we consider a subset of $M$ containing 0 and any one element from the rest say $k h$, where $1 \leq k \leq p$, then the set of mappings from
$E$ to $\{0, k h\}$ are the fuzzy subsets of $F$ which are different from crisp subsets. We denote this set of fuzzy subsets by $F_{k}$ and it is contained in $F$. The numbers of elements in $F_{k}$ is $2^{n}$, which are as follows:

$$
\begin{aligned}
& I_{0}=\varnothing=\left\{\left(x_{0}, 0\right),\left(x_{1}, 0\right), \ldots,\left(x_{n-2}, 0\right),\left(x_{n-1}, 0\right)\right\}, \\
& I_{1}=\left\{\left(x_{0}, 0\right),\left(x_{1}, 0\right), \ldots,\left(x_{n-2}, 0\right),\left(x_{n-1}, k h\right)\right\}, \\
& I_{2}=\left\{\left(x_{0}, 0\right),\left(x_{1}, 0\right), \ldots,\left(x_{n-2}, k h\right),\left(x_{n-1}, 0\right)\right\}, \\
& I_{3}=\left\{\left(x_{0}, 0\right),\left(x_{1}, 0\right), \ldots,\left(x_{n-2}, k h\right),\left(x_{n-1}, k h\right)\right\},
\end{aligned}
$$

$$
\begin{aligned}
& I_{n-2}=\left\{\left(x_{0}, k h\right),\left(x_{1}, k h\right), \ldots,\left(x_{n-2}, k h\right),\left(x_{n-1}, 0\right)\right\}, \\
& I_{n-1}=\left\{\left(x_{0}, k h\right),\left(x_{1}, k h\right), \ldots,\left(x_{n-2}, k h\right),\left(x_{n-1}, k h\right)\right\} .
\end{aligned}
$$

Hence, $F_{k}=\left\{I_{0}, I_{1}, I_{2} \ldots, I_{n-1}\right\}$ is the introduced family of fuzzy subsets. In $F_{k}$, the empty subset is denoted by $I_{0}$ or $\varnothing$ and this subset mathematically written as $\varnothing=\left\{\left(x_{0}, 0\right),\left(x_{1}, 0\right) \ldots\left(x_{n-1}, 0\right)\right\}$, which is similar to the empty subset of $F$. Again we get a fuzzy subset denoted by $I_{n-1}$, and which is mathematically written as $I_{n-1}=\left\{\left(x_{0}, k h\right),\left(x_{1}, k h\right), \ldots\left(x_{n-1}, k h\right)\right\}$ and this fuzzy subset is defined as the Universal fuzzy subset of $F_{k}$ which may be different from the universal set of $F$. The introduced families of fuzzy subsets denoted by $F_{k}$ and $F$ are explained in the following numerical example:

\subsection{Example}

Let $E_{1}=\left\{x_{0}, x_{1}, x_{2}\right\} \quad$ be a finite set and $M_{1}=\{0, h, 2 h, 3 h=1\}$ is the set of membership values, where $h=\frac{1}{3}$. Then the mappings from $E_{1}$ to $M_{1}$ will form a set $F$ of $4^{3}=64$ fuzzy subsets which are shown bellow. We denote these fuzzy subsets of $F$ by p-adic numbering as follows- 
$0=\left\{\left(x_{0}, 0\right),\left(x_{1}, 0\right),\left(x_{2}, 0\right)\right\}$,

where $0=(000)_{4}=0 \times 4^{2}+0 \times 4^{1}+0 \times 4^{0}$.

$1=\left\{\left(x_{0}, 0\right),\left(x_{1}, 0\right),\left(x_{2}, h\right)\right\}$,

where $1=(001)_{4}=0 \times 4^{2}+0 \times 4^{1}+1 \times 4^{0}$.

$2=\left\{\left(x_{0}, 0\right),\left(x_{1}, 0\right),\left(x_{2}, 2 h\right)\right\}$,

where $2=(002)_{4}=0 \times 4^{2}+0 \times 4^{1}+2 \times 4^{0}$.

$3=\left\{\left(x_{0}, 0\right),\left(x_{1}, 0\right),\left(x_{2}, 3 h\right)\right\}$,

where $3=(003)_{4}=0 \times 4^{2}+0 \times 4^{1}+3 \times 4^{0}$.

where, $63=(333)_{4}=3 \times 4^{2}+3 \times 4^{1}+3 \times 4^{0}$.

and 1,2,3 are the co-efficients of $h$.

Hence, $F=\{0,1,2,3, \ldots, 62,63\}$.

Now, if we consider a subset $M_{2}$ of $M_{1}$, such that $M_{2}=\{0,2 h\}$. Then the mappings from $E_{1}$ to $M_{2}$ are the fuzzy subsets. Let us denote this set of fuzzy subsets by $F_{3}$ whose elements are:

$0=\left\{\left(x_{0}, 0\right),\left(x_{1}, 0\right),\left(x_{2}, 0\right)\right\}$,

$2=\left\{\left(x_{0}, 0\right),\left(x_{1}, 0\right),\left(x_{2}, 2 h\right)\right\}$,

$8=\left\{\left(x_{0}, 0\right),\left(x_{1}, 2 h\right),\left(x_{2}, 0\right)\right\}$,

$10=\left\{\left(x_{0}, 0\right),\left(x_{1}, 2 h\right),\left(x_{2}, 2 h\right)\right\}$,

$32=\left\{\left(x_{0}, 2 h\right),\left(x_{1}, 0\right),\left(x_{2}, 0\right)\right\}$,

$34=\left\{\left(x_{0}, 2 h\right),\left(x_{1}, 0\right),\left(x_{2}, 2 h\right)\right\}$,

$40=\left\{\left(x_{0}, 2 h\right),\left(x_{1}, 2 h\right),\left(x_{2}, 0\right)\right\}$,

$42=\left\{\left(x_{0}, 2 h\right),\left(x_{1}, 2 h\right),\left(x_{2}, 2 h\right)\right\}$,

So, $F_{3}=\{0,2,8,10,32,34,40,42\}$. The numbers of elements in $F_{3}$ is $2^{3}=8$ since the set $E$ contains three elements, that is $|E|=3$.

\section{REDEFINITION OF THE COMPLEMENT OPERATION}

Now we are going to redefine the complement operation for the fuzzy subsets of $F_{k}$. We get that two fuzzy subsets $\underset{\sim}{a}$ and $\underset{\sim}{a}$ are complement of each other if, $\mu_{a^{\prime}}(x)=\left\{1-\mu_{\underset{\sim}{a}}(x)\right\}$.Now, we redefine this operation for the set $F_{k}$ of fuzzy subsets of the finite set $E$. This operation is redefined as: $\mu_{a^{\prime}}(x)=\left\{g-\mu_{a}(x)\right\}$, where $g=k h \in M$, where $1 \leq k \leq p$.

\subsection{Theorem: The fuzzy subsets of $F_{k}$ holds the Complement laws of Boolean algebra}

Proof: Let $\underset{\sim}{a} \in F_{k}$ be a fuzzy subset and $\underset{\sim}{a^{\prime}}$ be its complement such that,

$\underset{\sim}{a}=\left\{\left(x, \mu_{a}(x)\right): x \in E, \mu_{a}(x) \in\{0, g\}\right\}$.

Assuming, $\forall x \in E$,

$\min \left(\mu_{a}(x), \mu_{a^{\prime}}(x)\right)=\min \left[\left\{\mu_{a}(x), g-\mu_{a}(x)\right\}\right]$.

So, for every $x$ either $\mu_{a}(x)=0$ or $g-\mu_{a}(x)=0$.

$\therefore \underset{\sim}{a} \underset{\sim}{a}{ }^{\prime}=I_{0}=\varnothing=$ the empty subset.

Again,

$$
\begin{aligned}
\max \left(\mu_{a}(x), \mu_{a^{\prime}}(x)\right) & =\max \left[\mu_{a}(x), g-\mu_{a}(x)\right] \\
& =g .
\end{aligned}
$$

so, for every $x$ either $\mu_{a}(x)=g$ or g- $\mu_{a}(x)=g$.

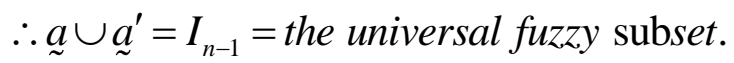

Hence, it is proved that the complement laws are hold by the fuzzy subsets of $F_{k}$.

\subsection{Example}

Let a finite set $E=\left\{x_{0}, x_{1}, x_{2}\right\} \quad$ and $M=\{0, h, 2 h, 3 h=1\}$ be the set of membership values, where $h=\frac{1}{3}$. Now considering a subset $N$ of $M$ so that $N=\{0, h\}$, then the mappings from $E$ to $N$ will contain $|N|^{|E|}=2^{3}=8$ fuzzy subsets as follows-

$0=\left\{\left(x_{0}, 0\right),\left(x_{1}, 0\right),\left(x_{2}, 0\right)\right\}$,

$1=\left\{\left(x_{0}, 0\right),\left(x_{1}, 0\right),\left(x_{2}, h\right)\right\}$,

$4=\left\{\left(x_{0}, 0\right),\left(x_{1}, h\right),\left(x_{2}, 0\right)\right\}$,

$5=\left\{\left(x_{0}, 0\right),\left(x_{1}, h\right),\left(x_{2}, h\right)\right\}$,

$16=\left\{\left(x_{0}, h\right),\left(x_{1}, 0\right),\left(x_{2}, 0\right)\right\}$,

$17=\left\{\left(x_{0}, h\right),\left(x_{1}, 0\right),\left(x_{2}, h\right)\right\}$,

$20=\left\{\left(x_{0}, h\right),\left(x_{1}, h\right),\left(x_{2}, 0\right)\right\}$,

$21=\left\{\left(x_{0}, h\right),\left(x_{1}, h\right),\left(x_{2}, h\right)\right\}$ 
Where, $0,1,4,5,16,17,20$ and 21 are the p-adic numbers and the set of all fuzzy subsets $F_{3}=\{0,1,4,5,16,17,20,21\}$,

Now, the complements of:

$$
\begin{aligned}
0 & =\left\{\left(x_{0}, h-0\right),\left(x_{1}, h-0\right),\left(x_{2}, h-0\right)\right\} \\
& =\left\{\left(x_{0}, h\right),\left(x_{1}, h\right),\left(x_{2}, h\right)\right\} \\
& =21, \\
1 & =\left\{\left(x_{0}, h-0\right),\left(x_{1}, h-0\right),\left(x_{2}, h-h\right)\right\} \\
& =\left\{\left(x_{0}, h\right),\left(x_{1}, h\right),\left(x_{2}, 0\right)\right\} \\
& =20 .
\end{aligned}
$$

Hence, we get that $0,1,4,5$ are the complements of $21,20,17$ and16 respectively.

Now,

$0 \cup 21=1 \cup 20=4 \cup 17=5 \cup 16=21$

= the universal fuzzy subset.

Again,

$0 \cap 21=1 \cap 20=4 \cap 17=5 \cap 16=0=\varnothing$

= the empty subset.

Hence, the complement laws hold.

\subsection{Theorem: The elements (fuzzy subsets) of $F_{k}$ forms a Boolean lattice.}

Proof: The relation ' $\subseteq$ '(inclusion) defined on $F_{k}$ is reflexive, antisymmetric and transitive since the elements of $F_{k}$ are in point-wise ordering of real numbers [5]. Hence, $\left(F_{k}, \subseteq\right)$ is a po-set.

Again, let $\underset{\sim}{A}, \underset{\sim}{B} \in F_{k}$ are two fuzzy subsets, where,

$\underset{\sim}{A}=\left\{\left(x, \mu_{\sim}(x)\right\}, \mu_{\sim}(x) \in\{0, g\}\right.$,

$\underset{\sim}{B}=\left\{\left(x, \mu_{B}(x)\right\}, \mu_{\sim}(x) \in\{0, g\}\right.$,

where, $g=k h$, and $1 \leq k \leq p$.

Now, the intersection and union of the two fuzzy subsets $\underset{\sim}{A}$ and $\underset{\sim}{B}$,

$$
\begin{aligned}
& \mu_{\underset{\sim}{A \cap B}}(x)=\min \left(\mu_{\sim}(x), \mu_{\sim_{B}}(x)\right) \\
& =\mu_{A}(x) \wedge \mu_{B}(x) \text {. } \\
& \mu_{\underset{\sim}{A \cup B}}(x)=\max \left(\mu_{A}(x), \mu_{\sim_{B}}(x)\right) \\
& =\mu_{A}(x) \vee \mu_{\sim_{B}}(x) \text {. }
\end{aligned}
$$

So, $\underset{\sim}{A} \wedge \underset{\sim}{B}$ and $\underset{\sim}{A} \vee \underset{\sim}{B}$ exist in $\left(F_{k}, \subseteq\right)$ as we have from the definition of union and intersection. Also, the set $F_{k}$ is closed under two binary operations union and intersection. Hence, $\left(F_{k}, \subseteq\right)$ is a lattice under the binary operations union and intersection. We know that fuzzy subsets hold distributive law. Hence $\left(F_{k}, \subseteq\right)$ is a distributive lattice.

Again, it is a bounded lattice because for any fuzzy subset $\underset{\sim}{A} \in\left(F_{k}, \subseteq\right)$ there exists a fuzzy subset $\underset{\sim}{D} \subseteq \underset{\sim}{A}$. This $\underset{\sim}{D}$ is defined as the empty subset of $E$ such that $\mu_{D}(x)=0, \forall x \in E$. This $\underset{\sim}{D}$ can be called as the 'bottom element' (infimum) and denoted as ' 0 '. On the other hand, $\forall \underset{\sim}{A} \in\left(F_{k}, \subseteq\right)$ there exists $\underset{\sim}{D^{\prime}}$ such that $\underset{\sim}{A} \subseteq \underset{\sim}{D^{\prime}}$. This $\underset{\sim}{D^{\prime}}$ is defined as the universal fuzzy subset of $E$ such that $\mu_{D^{\prime}}(x)=g \forall x \in E$. This subset can be called as the 'top element' (supremum) and denoted by ' $g$ ' . Hence, the lattice is distributive and bounded. Also we have proved that the lattice is complemented since each fuzzy subset has its complement. Hence, $\left(F_{k}, \subseteq\right)$ is a Boolean lattice.

\subsection{Theorem: The fuzzy subsets of $F_{k}$ forms a Boolean algebra.}

Proof: We have the set $F_{k}$ of fuzzy subsets of a finite set discussed in above. Then structure $B=\left(F_{k}, \square,+, /, 0, g\right)$, where $\square$ and ' + 'represents two binary operations intersection and union respectively, '/ represents a unary operation complementation and two distinct elements ' $\mathbf{0}$ ' (bottom element) and 'g' (top element) forms a Boolean algebra since $F_{k}$ is a complemented distributive lattice.

\subsection{Proposition: The relation between the complement of a fuzzy subset in $F$ and the complement of the same element in $F_{k}$ is that the difference between their membership values is always equal to $p h-k h$.}

Proof: We can prove this proposition with the help of a numerical example. If we consider the numerical example 4.2 as illustrated above then the complements of the elements in $F_{3}$ and complements of the same elements in $F$ are shown in the following table and we can notice that the difference between the membership values of these two complements is $p h-k h$, where $p$ is any prime number and $h=\frac{1}{p}$ for $1 \leq k \leq p$. In this case, which is $3 h-h=2 h$. 
Table 1. Relation between the two kinds of complements of the same elements (showing only the membership values)

\begin{tabular}{|c|c|c|c|}
\hline $\begin{array}{c}\text { Elements in } \\
F_{3}\end{array}$ & $\begin{array}{c}\text { Complement } \\
\text { in } F_{3}\end{array}$ & $\begin{array}{c}\text { Complement } \\
\text { in } F\end{array}$ & Difference \\
\cline { 1 - 2 }$(0,0,0)$ & $(h, h, h)$ & $(3 h, 3 h, 3 h)$ & \\
\cline { 1 - 2 }$(0,0, h)$ & $(h, h, 0)$ & $(3 h, 3 h, 2 h)$ & \multirow{2}{*}{$\begin{array}{c}2 h \\
(3 h-h)\end{array}$} \\
\cline { 1 - 2 }$(0, h, 0)$ & $(h, 0, h)$ & $(3 h, 2 h, 3 h)$ & \\
\cline { 1 - 2 }$(0, h, h)$ & $(h, 0,0)$ & $(3 h, 2 h, 2 h)$ & \\
\end{tabular}

\section{SUBSET OF A FUZZY SUBSET, FUZZY POWER SET OF A SUBSET (for the introduced fuzzy subsets)}

This section introduces the definition of the subset of a fuzzy subset and then the fuzzy power set of a subset. For a fuzzy subset $\underset{\sim}{S}$, written as follows, where $\mu\left(x_{i}\right)$ are the membership values of the element $x_{i}$, for $i=0,1 \ldots, n-1$.

$\underset{\sim}{S}=\left\{\frac{\mu_{S}\left(x_{0}\right)}{x_{0}}+\frac{\mu_{S}\left(x_{1}\right)}{x_{1}}+\ldots . . \frac{\mu_{S_{n}}\left(x_{n-2}\right)}{x_{n-2}}+\frac{\mu_{S_{S}}\left(x_{n-1}\right)}{x_{n-1}}\right\}$,

we can get $2^{m}$ subsets, where $m$ =numbers of elements with non-zero membership. They are:

${\underset{\sim}{S}}_{0}=\varnothing=\left\{\frac{0}{x_{0}}+\frac{0}{x_{1}}+\ldots, \frac{0}{x_{n-2}}+\frac{0}{x_{n-1}}\right\}$,

$\underset{\sim}{S_{1}}=\left\{\frac{0}{x_{0}}+\frac{0}{x_{1}}+\ldots, \frac{0}{x_{n-2}}+\frac{\mu_{\underline{\sim}}\left(x_{n-1}\right)}{x_{n-1}}\right\}$,

${\underset{\sim}{S}}_{2}=\left\{\frac{0}{x_{0}}+\frac{0}{x_{1}}+\ldots, \frac{\mu_{S}\left(x_{n-2}\right)}{x_{n-2}}+\frac{\mu_{S}\left(x_{n-1}\right)}{x_{n-1}}\right\}$,

$\underset{\sim}{S_{n-2}}=\left\{\frac{\mu_{S}\left(x_{0}\right)}{x_{0}}+\frac{\mu_{S}\left(x_{1}\right)}{x_{1}}+\ldots, \frac{\mu_{S}\left(x_{n-2}\right)}{x_{n-2}}+\frac{0}{x_{n-1}}\right\}$,

$\underset{\sim}{S_{n-1}}=\left\{\frac{\mu_{S}\left(x_{0}\right)}{x_{0}}+\frac{\mu_{S}\left(x_{1}\right)}{x_{1}}+\ldots, \frac{\mu_{S}\left(x_{n-2}\right)}{x_{n-2}}+\frac{\mu_{S}\left(x_{n-1}\right)}{x_{n-1}}\right\}$.

This is explained in the following numerical examples-

\subsection{Example}

If $\underset{\sim}{A}=\left\{\left(x_{0}, 0.1\right),\left(x_{1}, 0.2\right),\left(x_{2}, 0.3\right)\right\}$ is a fuzzy subset then it has $2^{3}=8$ subsets as follows-

$\underset{\sim 0}{A_{0}}=\varnothing=\left\{\left(x_{0}, 0\right),\left(x_{1}, 0\right),\left(x_{2}, 0\right)\right\}$,

$\underset{\sim}{A}=\left\{\left(x_{0}, 0\right),\left(x_{1}, 0\right),\left(x_{2}, 0.3\right)\right\}$,

$\underset{\sim 2}{A_{2}}=\left\{\left(x_{0}, 0\right),\left(x_{1}, 0.2\right),\left(x_{2}, 0\right)\right\}$,

$\underset{\sim 3}{A_{3}}=\left\{\left(x_{0}, 0.1\right),\left(x_{1}, 0\right),\left(x_{2}, 0\right)\right\}$,

$\underset{\sim}{A_{4}}=\left\{\left(x_{0}, 0\right),\left(x_{1}, 0.2\right),\left(x_{2}, 0.3\right)\right\}$,

$A_{5}=\left\{\left(x_{0}, 0.1\right),\left(x_{1}, 0\right),\left(x_{2}, 0.3\right)\right\}$,

$\underset{\sim 6}{A_{6}}=\left\{\left(x_{0}, 0.1\right),\left(x_{1}, 0.2\right),\left(x_{2}, 0\right)\right\}$,

$A_{7}=\left\{\left(x_{0}, 0.1\right),\left(x_{1}, 0.2\right),\left(x_{2}, 0.3\right)\right\}$.

\subsection{Example}

If $\underset{\sim}{A}=\left\{\left(x_{0}, 0\right),\left(x_{1}, 0.2\right),\left(x_{2}, 0.3\right)\right\}$ is a fuzzy subset then it has $2^{2}=4$ subsets as follows-

$\underset{\sim 0}{A_{0}}=\varnothing=\left\{\left(x_{0}, 0\right),\left(x_{1}, 0\right),\left(x_{2}, 0\right)\right\}$,

$\underset{\sim 1}{A}=\left\{\left(x_{0}, 0\right),\left(x_{1}, 0\right),\left(x_{2}, 0.3\right)\right\}$,

$\underset{\sim 2}{A_{2}}=\left\{\left(x_{0}, 0\right),\left(x_{1}, 0.2\right),\left(x_{2}, 0\right)\right\}$,

$A_{\sim}=\left\{\left(x_{0}, 0\right),\left(x_{1}, 0.2\right),\left(x_{2}, 0.3\right)\right\}$.

\subsection{Definition of fuzzy power set of a subset}

Now we are going to redefine the Fuzzy Power Set for a subset of a fuzzy subset of $F_{k}$.

For any fuzzy set $\underset{\sim}{S}$, we define the fuzzy power set of $\underset{\sim}{S}$ as the set of all the subsets (as per our definition of subset) of $\underset{\sim}{S}$, including the empty subset and the fuzzy subset $\underset{\sim}{S}$ itself and we denote it as $P(\underset{\sim}{S})$. The number of elements in $P(\underset{\sim}{S})$ is $2^{m}$ where $m=$ numbers of element with non-zero membership.

\subsection{Example}

If $\underset{\sim}{A}=\left\{\left(x_{0}, 0.1\right),\left(x_{1}, 0.2\right),\left(x_{2}, 0.3\right)\right\}$ is a fuzzy subset then it has $2^{3}=8$ subsets as explained in the example 5.1 since it has three elements with non-zero membership which are: $\underset{\sim 0}{A}, \underset{\sim}{A}, \underset{\sim 2}{A}, \underset{\sim 3}{A_{3}}, \underset{\sim 4}{A_{4}}, \underset{\sim 5}{A_{5}}, \underset{\sim 6}{A_{6}}$ and $\underset{\sim 7}{A_{7}}$.

$\therefore$ The power set of $\underset{\sim}{A}, P(\underset{\sim}{A})=\left\{A_{0}, A_{1}, \ldots \ldots . A_{7}\right\}$. 


\subsection{Proposition: $F_{k}$ can be expressed as a power set of the fuzzy subset $I_{n-1}$}

Proof: From the definition of fuzzy power set we can get that

$$
I_{n-1}=\left\{\left(x_{0}, k h\right),\left(x_{1}, k h\right) \ldots,\left(x_{n-2}, k h\right),\left(x_{n-1}, k h\right)\right\}
$$

will contain $2^{n}$ elements since the number of elements with non-zero membership is $n$. They are as follows-

$$
\begin{aligned}
& \therefore P\left(I_{n-1}\right)= \\
& {\left[I_{0}=\varnothing=\left\{\left(x_{0}, 0\right),\left(x_{1}, 0\right), \ldots,\left(x_{n-2}, 0\right),\left(x_{n-1}, 0\right)\right\},\right.} \\
& I_{1}=\left\{\left(x_{0}, 0\right),\left(x_{1}, 0\right) \ldots,\left(x_{n-2}, 0\right),\left(x_{n-1}, k h\right)\right\}, \\
& I_{2}=\left\{\left(x_{0}, 0\right),\left(x_{1}, 0\right) \ldots,\left(x_{n-2}, k h\right),\left(x_{n-1}, 0\right)\right\}, \\
& I_{3}=\left\{\left(x_{0}, 0\right),\left(x_{1}, 0\right) \ldots,\left(x_{n-2}, k h\right),\left(x_{n-1}, k h\right)\right\},
\end{aligned}
$$

$$
I_{n-2}=\left\{\left(x_{0}, k h\right),\left(x_{1}, k h\right) \ldots,\left(x_{n-2}, k h\right),\left(x_{n-1}, 0\right)\right\},
$$$$
\left.I_{n-1}=\left\{\left(x_{0}, k h\right),\left(x_{1}, k h\right) \ldots,\left(x_{n-2}, k h\right),\left(x_{n-1}, k h\right)\right\}\right] \text {. }
$$

Hence, $P\left(I_{n-1}\right)=F_{k}=\left\{I_{0}, I_{1}, I_{2} \ldots, I_{n-1}\right\}$.

\section{CONCLUSIONS}

This paper has introduced a kind of family of fuzzy subsets which forms Boolean algebra. These concepts can lead to a new direction and there is a lot of potential growth in this direction.

\section{ACKNOWLEDGMENTS}

The authors would like to express their sincere thanks to the referees for their valueable comments and suggestions which help a lot for improving the presentation of this paper.

\section{REFERENCES}

[1] G. J. Klir, B. Yuan, Fuzzy Sets and Fuzzy Logic: Theory and Applications, Prentice Hall, Englewood Cliffe, NJ, 1995.

[2] G. Bojadziev, M. Bojadziev, Fuzzy Sets, Fuzzy Logic, Applications: Advances in Fuzzy Systems, World Scientific, vol. 5.

[3] L. A., Zadeh, Fuzzy sets, Information and Control, 8 (1965) 338-353.

[4] G. Birkhoff, Lattice theory, American Mathematical Society, Providence, RI, 1984.

[5] V. Murali, and B. B. Makamba, Finite Fuzzy Sets, Int. J. Gen. Systems, 34 (2005) 61-75.

[6] M. Dhar, Fuzzy Sets towards Forming Boolean Algebra, International Journal of energy, Information and Communication, vol. 2, issue 4, Nov. 2011.

[7] T. J. Neog, D. K. Sut, Complement of an Extended Fuzzy Set, IJCA (0975-8887), Volume 29-No 3, Sep. 2011.

[8] B. Seselja, A. Tepavcevic, Lattice and Poset Valued fuzzy structures-theory and applications, University of Novi Sad, Ser., August 20, 2009. 